\title{
Robótica quirúrgica, desde los grandes asistentes hasta la nanotecnología
}

\author{
Surgical robotics: from the great assistants to nanotechnology
}

\author{
María Cristina Chaparro Velasco, Óscar Andrés Vivas Albán \\ Ingeniera en Electrónica y Telecomunicaciones, Grupo en Automática Industrial AI, Universidad del Cauca, \\ Popayán, Colombia \\ Correo-e: cristinach@unicauca.edu.co, avivas@unicauca.edu.co
}

\begin{abstract}
Resumen-En este artículo se hace una revisión del desarrollo de la robótica quirúrgica empezando por los primeros robots industriales utilizados en procedimientos médicos, siguiendo con los grandes asistentes robóticos para cirugía abdominal, los mini y micro robots, sus tipos y diferentes aplicaciones, y finalmente se muestran los nano robots diseñados para aplicaciones quirúrgicas. El objetivo es mostrar una visión general de las últimas actualizaciones en cirugía robótica y las potenciales aplicaciones de esta $y$ de nuevas tecnologías en diferentes procedimientos quirúrgicos, además de exponer las ventajas y desventajas de los diferentes sistemas robóticos.
\end{abstract}

Palabras Clave - Robots quirúrgicos, mini robots, micro robots, nano robots, robótica médica.

Abstract- This article reviews the development of surgical robotics, starting with the first industrial robots used in medical procedures, following the major robotic assistants for abdominal surgery, mini and micro robots, their types and different applications, and finally the designed surgical nano-robots. The aim is to show an overview of the latest updates on robotic surgery and the potential applications of this, and new technologies on different surgical procedures, in addition to exposing the advantages and disadvantages of different robotic systems.

Key Word-Medical robotics, micro-robots, mini robots, surgical robots.

\section{INTRODUCCIÓN}

Este documento presenta una investigación detallada sobre los últimos avances realizados en el diseño, construcción y pruebas de los robots quirúrgicos y sus diferentes aplicaciones. Se resaltan los beneficios de la utilización de robots quirúrgicos y las ventajas que presentan los mini o micro robots en diferentes procedimientos.

El desarrollo de nuevas técnicas de cirugía ha abierto el camino para que los médicos acepten el uso de sistemas robóticos durante los procedimientos, sin embargo, al ser un tema muy reciente, es necesario dar a conocer el estado actual de estas nuevas tecnologías para incentivar su desarrollo y uso en los campos de la ingeniería y la medicina.

\section{ANTECEDENTES DE LOS ROBOTS QUIRÚRGICOS}

Durante los últimos años, la medicina ha evolucionado rápidamente para generar beneficios tanto a pacientes como a médicos. Entre los avances más reconocidos se destaca la MIS (cirugía mínimamente invasiva, por sus siglas en inglés), un recurso que se creó para evitar realizar incisiones grandes y profundas en el cuerpo de los pacientes. Esta técnica está ampliamente difundida y entre sus ventajas se encuentra la diminución del tiempo de recuperación del paciente, reducción del riesgo de hemorragias y menores costos hospitalarios [1]. El tipo de cirugías que se realizan con esta técnica son muy variadas y entre estas se destaca la laparoscopia, que se ha utilizado en pacientes de todas las edades. Pero también tiene varias desventajas como la entrega de visión en solo dos dimensiones, poco sentido de profundidad, y posiciones muy incómodas y tensionantes para el cirujano [1] [2].

Con el progreso de la MIS aparecieron nuevos avances en cirugía que permitieron desarrollar otros tipos de procedimientos como NOTES y LESS.

NOTES (cirugía endoscópica transluminal por orificios naturales, por sus siglas en inglés) es un procedimiento menos invasivo que la laparoscopia, pues es realizado a través de orificios naturales (boca, vagina, recto, uretra) y tiene muchas aplicaciones como peritoneoscopia transgástrica y transvesical, ligadura de trompas transvaginal, histerectomía y colecistectomía [3]. Entre las ventajas de este tipo de cirugía se destaca la no existencia de cicatrices, reducción del dolor, menos complicaciones, carácter ambulatorio y menor tiempo 
de recuperación [4], sin embargo también presenta varias desventajas como la necesidad de utilizar instrumentos costosos, riesgo de perforar otros órganos en el camino, hemorragias [5], infecciones y visibilidad limitada [4].

Un procedimiento intermedio entre MIS y NOTES es la laparoendoscopia por puerto único, la cual se realiza como su nombre lo indica, a través de una única incisión abdominal, por donde ingresa cámara e instrumentos quirúrgicos. Además de las ventajas que ya tiene la laparoscopia tradicional, LESS (como se conoce en inglés) presenta menor daño al tener menos incisiones, disminución del dolor, rápida recuperación y mejor aspecto estético, sin embargo introduce nuevos retos ya que produce disminución de la triangulación, transposición y colisión de los instrumentos quirúrgicos y vista en línea de estos, lo que disminuye la habilidad del cirujano al complicar un procedimiento que con otra técnica puede ser muy sencillo [6].

Ante los avances e inconvenientes presentados por estas técnicas la robótica ha ofrecido varias soluciones. La primera fue la utilización de robots industriales como asistentes en las cirugías. Así, el robot industrial PUMA 560 se utilizó en 1985 en el campo de la neurocirugía [2] [7], convirtiéndose en el primer robot quirúrgico, aunque no fue diseñado originalmente para este propósito. Entre los primeros asistentes quirúrgicos se encuentra Probot, un sistema guiado por ultrasonido para realizar extirpación total de la próstata [8] y Robodoc, que en 1992 fue aprobado para realizar reemplazo total de cadera [9]. Estos grandes asistentes hacen parte de lo que algunos autores llaman la "primera generación" de plataformas robóticas para cirugía [10] y sus principales desventajas estaban en que contaban con interfaces básicas y limitadas, y la falta de un sistema de diagnóstico que permitiera saber dónde iba a estar ubicado el robot dentro del cuerpo humano, lo que generaba largas reuniones para planear la operación.

\section{GRANDES ASISTENTES ROBÓTICOS}

En los últimos 30 años se han diseñado, probado y comercializado diversos robots quirúrgicos que han servido como asistentes en varios tipos de procedimientos como cirugía general, ortopédica, urológica, etcétera [11].

Los sistemas robóticos para cirugía desarrollados después de la mencionada primera generación tienen una configuración maestro-esclavo y están conformados por la consola del cirujano, que será el maestro que controlará los brazos robóticos (esclavos) que realizarán la cirugía. Bajo estos preceptos apareció y fue aprobado en 1994 el sistema endoscópico automático para posicionamiento óptimo Aesop, un brazo robótico para guiar una cámara laparoscópica, controlada por comandos de voz [12]. Fue el primero en ser utilizado en cirugías robóticas abdominales y fue construido por la empresa californiana Computer Motion. Esta empresa fue la desarrolladora en 1998 del sistema Zeus, un robot compuesto por una consola para el cirujano, un sistema de video tridimensional y tres brazos robóticos para realizar la cirugía, uno de ellos era el Aesop [13].

Paralelamente al desarrollo del Zeus, la empresa Intuitive Surgical creó el Da Vinci Surgical System [14] que fue aprobado para su uso comercial en el año 2000. En 2003 esta empresa y Computer Motion se fusionan [2] suspendiendo el desarrollo del Zeus y enfocando todos sus esfuerzos tecnológicos en el mejoramiento del Da Vinci, creando así el mejor sistema robótico para cirugía que se conoce hasta el momento y generando un monopolio en el mercado de los asistentes robóticos para cirugía. Esta situación se ha mantenido hasta la fecha [10].

El Da Vinci Surgical System tiene cuatro componentes característicos: la consola de mando, el soporte con brazos robóticos, instrumentos específicos para cirugía y un sistema de visión [15]. La consola de mando es desde donde el cirujano controla los brazos robóticos, tiene un sensor infrarrojo que detecta cuando el cirujano se sienta para empezar la operación, permite que el cirujano se mantenga sentado durante todo el procedimiento y contiene controles y pedales para manipular los instrumentos. El soporte para brazos robóticos es una base que se puede desplazar por la sala para acomodarla como más convenga en la cirugía, puede sostener hasta cuatro brazos robóticos que son los que replican los movimientos del cirujano y manipulan la cámara y los instrumentos. Estos tienen siete grados de movimiento para seguir los movimientos de la mano y la muñeca del médico y pueden cauterizar, suturar o manipular diversos tejidos. El sistema de visión es de alta resolución, tiene opción de acercarse hasta 10 veces, entrega imágenes en 3D y tiene una potente iluminación lo que permite navegación estable y precisa durante los procedimientos [2] [10] [15] [14]. Las últimas actualizaciones del Da Vinci, realizadas en 2014, han decantado en un sistema menos voluminoso y más ergonómico. Sin embargo, este sistema sigue teniendo problemas por su gran tamaño, la falta de realimentación al cirujano durante los procedimientos y el desmontaje que se hace necesario durante algunos procedimientos, lo cual aumenta la duración de la cirugía y por lo tanto de la anestesia suministrada al paciente [15].

Recientemente se han empezado a desarrollar otros sistemas robóticos que pueden ser competencia para el Da Vinci. Estos se han basado en una arquitectura similar a este como es el caso de Amadeus, desarrollado por la canadiense Titan Medical. Sin embargo por problemas de infracción de patente se detuvo su desarrollo, lo que llevó a esta empresa a desarrollar el Sport, un robot quirúrgico para realizar operaciones por puerto único como la LESS y que tiene un costo mucho menor que el Da Vinci. La empresa ha obtenido dos patentes y se espera su comercialización desde 2016 [16] [10]. La italiana Sofar ha estado desarrollando el sistema Telelap Alf-X [17], el cual ha mejorado varios de los aspectos del Da Vinci pues tiene tecnología para la realimentación de fuerza, lo que genera una percepción realista, permite navegar siguiendo el movimiento de los ojos, despliega el campo de cirugía en 3D en una pantalla de cristal y permite el cambio de brazos robóticos si el cirujano mira un icono específico. La empresa Avra de Nueva York está desarrollando un sistema 
robótico quirúrgico modular o ASRS, por sus siglas en inglés. La modularidad de este sistema permite mayor libertad y adaptación a diferente procedimientos [10]. En Alemania, el Instituto de Robótica y Mecatrónica (RMC) junto con el Centro Aeroespacial Alemán (DLR), han desarrollado el DLR MiroSurge, un sistema robótico para cirugía compuesto de tres robots cirujanos Miro de siete grados de libertad. El sistema posee realimentación de fuerza, es pequeño, se puede manipular fácilmente $\mathrm{y}$ se ha utilizado sobre todo para investigación en tele cirugía. Además fue diseñada para este robot una consola bimanual háptica de seis grados de libertad, ideal para realizar suturas y disminuir la fatiga del cirujano. Últimamente este sistema se está adaptando para la futura realización de cirugía cardiaca, un tema nuevo en el área de la cirugía asistida por robots [18] [19] (Figura 1). De otra parte la Universidad Técnica de Eindhoven en los Países Bajos, ha desarrollado y patentado el sistema quirúrgico Sofie. Este es un asistente compacto, montado sobre la camilla, de relativo bajo costo, con ocho grados de libertad y capaz de operar un órgano desde diversos ángulos [20] [21] (Figura 2).

Todos estos sistemas sin embargo no han sido aún aprobados para su uso comercial lo que hace que el Da Vinci siga manteniendo su puesto privilegiado en el mercado de la cirugía robótica.

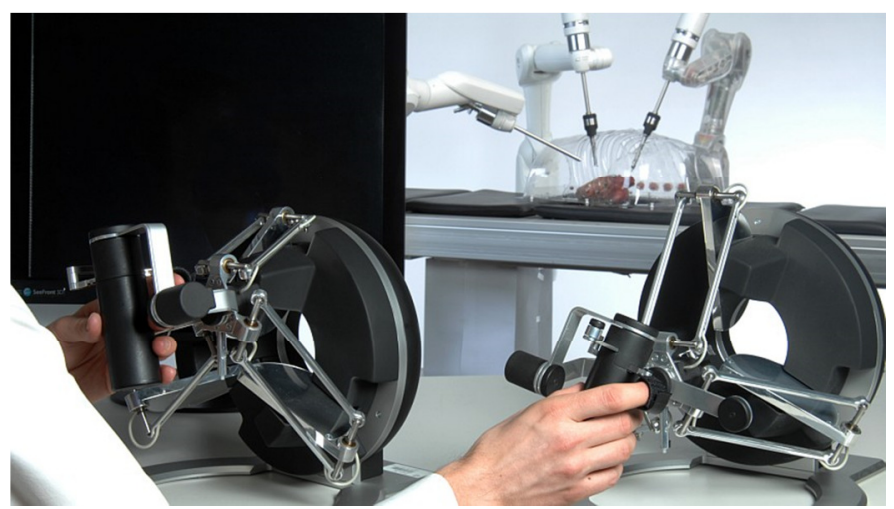

Figura 1. Robot MiroSurge del DLR, Alemania

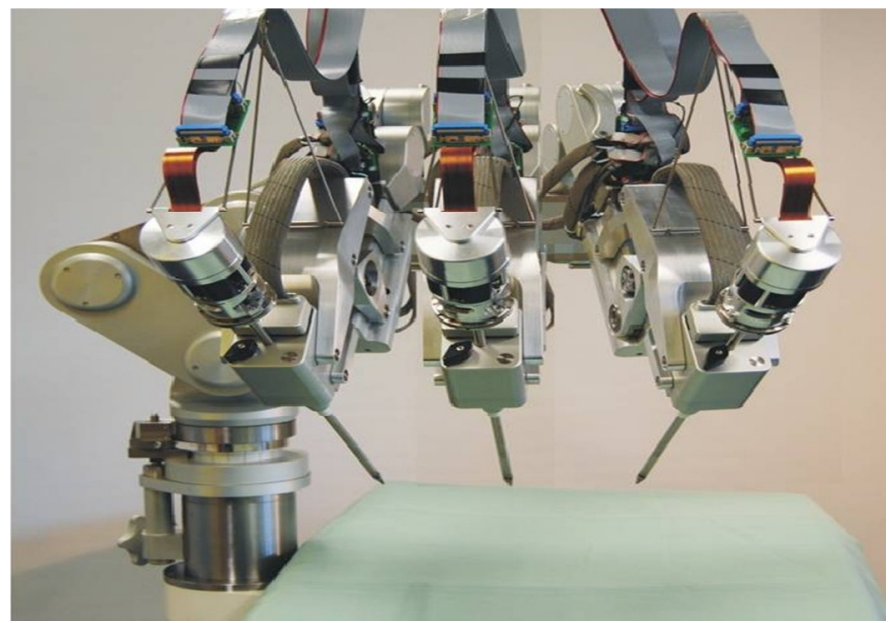

Figura 2. Robot SOFIE de la Universidad Eindhoven, Países Bajos

La utilización de grandes asistentes robóticos se ha visto ligada al desarrollo económico de los países. Esto se evidencia en la cantidad de robots que funcionan, tanto en asistencia como en entrenamiento, en cada país. Así hay más de 1000 sistemas Da Vinci en Estados Unidos y Canadá mientras en Latinoamérica hay solo 12 [22].

Colombia es uno de los pocos países de Latinoamérica en el cual se realizan cirugías robóticas y entrenamientos para aprender a manipular el robot Da Vinci [23] [24]. Esto sitúa al país en la vanguardia de la cirugía robótica y abre las puertas a la investigación en este tema. Dentro de los desarrollos nacionales se destaca el control de un brazo robótico a través del sensor Kinect de Microsoft y su potencial beneficio en el uso en cirugía robótica desarrollado en la Universidad Autónoma del Caribe [25]. En el 2010 en la Universidad Pontificia Bolivariana de Medellín se realizó un estudio para analizar la fuerza y el torque en la cirugía robótica mínimamente invasiva y presentó modelos matemáticos para calcular las fuerzas y el torque, esto con el fin de buscar una solución a uno de los principales problemas de las cirugías robóticas como es la falta de realimentación de fuerza y tacto [1]. En la Universidad del Cauca se han desarrollado diversos proyectos en robótica quirúrgica como el diseño y construcción de dos robots para cirugía laparoscópica: el LapBot [26] para realizar el procedimiento y el robot porta endoscopio Hibou [27]. También se ha implementado un simulador quirúrgico con robots [28], se diseñó una interfaz háptica para realizar procedimientos quirúrgicos que cuenta con realimentación de fuerza [29], se diseñó y construyó un guante háptico con realimentación vibratoria que sigue fielmente los movimientos de la mano real [30], y utilizando el Kinect de Microsoft se desarrolló un sistema para capturar movimientos que manipulen robots quirúrgicos virtuales [31].

\section{MicRo RoBOtS EN LA MEDICINA}

El campo de estudio de la robótica en miniatura es la micro robótica. Como su nombre lo indica, el estudio se centra en robots de tamaño y dimensiones menores a un milímetro o en aquellos que pueden manipular objetos milimétricos. El nombre micro robot se ha ampliado a todo robot de tamaño pequeño pero de acuerdo a un tamaño específico también se puede hablar de mini robots. Los robots miniatura empezaron a ser estudiados hace relativamente poco tiempo gracias a la aparición del microcontrolador que permitió la miniaturización de diferentes equipos electrónicos. Esto permitió que la robótica en miniatura tuviera un desarrollo vertiginoso en los últimos años al disminuir los costos de fabricación. Así, cada vez más grupos de investigación han enfocado sus esfuerzos en diseñar y construir pequeños robots que puedan utilizarse en aplicaciones militares, de construcción, vigilancia y en el campo de la salud. Los robots miniatura pueden ser de varios tipos: terrestres, nadadores, voladores y de enjambre (que puede ser conformado por cualquiera de los tipos indicados), y 
dependiendo de sus características físicas se podrían utilizar en los diferentes campos de estudio mencionados [32] [33].

En medicina, aunque los grandes asistentes robóticos quirúrgicos han representado múltiples avances en la cirugía laparoscópica, aún muestran inconvenientes relacionados con su gran tamaño, su manipulación, el difícil acceso a algunas partes del cuerpo humano y su alto costo. Mirando la evolución de la tecnología hacia el futuro existen muchos vectores de desarrollo que reemplazarán o complementarán los robots asistenciales. Entre estos se plantea el uso de micro robots para solucionar los problemas de acceso a lugares complejos en las cirugías, mejorar los tratamientos contra el cáncer y las biopsias, y disminuir la incomodidad, el dolor y el tiempo de recuperación del paciente [34].

En varios centros de investigación alrededor del mundo diversos mini robots biomiméticos enfocados en asistencia y tratamientos médicos han sido desarrollados, tomando como referencia gusanos, bacterias, espermatozoides o sanguijuelas [35] [36]. También se han considerado y desarrollado robots en forma de pastillas, cuyo principal inconveniente es la falta de control sobre sus movimientos [37]. Varios de estos mini robots han sido probados en animales mostrando sus múltiples ventajas [38], sin embargo aún no han sido aprobados para ser utilizados en humanos.

Los esfuerzos actuales están enfocados en diseñar mini robots aptos para funcionar en un ambiente hostil como es el cuerpo humano, puesto que uno de los mayores obstáculos de esta área es la locomoción dentro de un organismo vivo [39].

Otro problema a tener en cuenta es el control de los movimientos de un mini robot dentro un ser vivo. La solución más innovadora y reciente es el uso de campos magnéticos (imán externo que guía al mini robot interno) para tener un mayor control sobre estos, disminuir aún más su tamaño y evitar gastos extras en actuadores u otros elementos [32].

$\mathrm{Al}$ respecto se han venido desarrollando y se han propuesto múltiples tipos de mini robots magnéticos, que pretenden ser una solución a problemas como la falta de espacio en el área abdominal al realizar los procedimientos quirúrgicos [40], y la generalización de cirugías abdominales por una sola entrada o por orificios naturales. Dependiendo del tipo de procedimiento que asistan, estos mini robots magnéticos pueden ser tipo cámara [41][42], pinza [43], fuente de iluminación [42] o dispositivos para realizar biopsias [37]. Sus diseños se han basado en la tecnología de anclaje magnético [44], la cual ha permitido el diseño de instrumentos que pueden ser manipulados con magnetos externos al cuerpo sin generar daño a los tejidos del paciente [38], y además su utilización ha generado varias ventajas frente a procedimientos convencionales como la laparoscopia [45].

Una de las tecnologías de anclaje magnético más utilizadas en procedimientos quirúrgicos como LESS y NOTES es el sistema de guía y anclaje magnético MAGS [40]. Este sistema puede soportar diversos instrumentos, como cámaras, luces, pinzas, cauterizadores, pequeñas herramientas para mover órganos sin dañarlos y también instrumentos desplegables, los cuales han servido de base para el diseño de mini robots [46]. También mejora la triangulación, la visibilidad, ergonomía y la carga de trabajo percibida por los cirujanos [6]. Entre sus desventajas se encuentra la curva de aprendizaje lenta que tiene pues es una tecnología reciente y aún requiere de muchos experimentos para mejorar la práctica y la experiencia del médico. Este sistema, además de mejorar las técnicas de LESS y NOTES y revolucionar el diseño de instrumentos quirúrgicos, ha permitido la introducción al campo quirúrgico de los robots miniatura, al ser una posible solución para su manipulación dentro del cuerpo humano [41].

Los robots miniatura en la medicina se pueden utilizar para asistir cirugías o para diagnosticar. Han sido concebidos para corregir problemas de visibilidad y accesibilidad.

En este grupo se destacan las cápsulas endoscópicas, diseñadas para facilitar el procedimiento de la endoscopia, obtener datos más confiables y disminuir la incomodidad del paciente [34]. La cápsula endoscópica más conocida hasta el momento es la PillCam. Comercializada por Given Imaging, se trata de una video cápsula que recorre el intestino tomando y enviando imágenes en tiempo real. Está compuesta por una cápsula que se puede ingerir, un registrador de datos con sensores y cámara, y una estación de trabajo. Puede adquirir imágenes por ambos lados a una tasa de cuatro marcos por segundo [47] y en sus versiones más recientes se ha mejorado el ángulo de visión y la calidad de las imágenes que toma (Figura 3) [48]. Su principal desventaja es la falta de control de sus movimientos y velocidad.
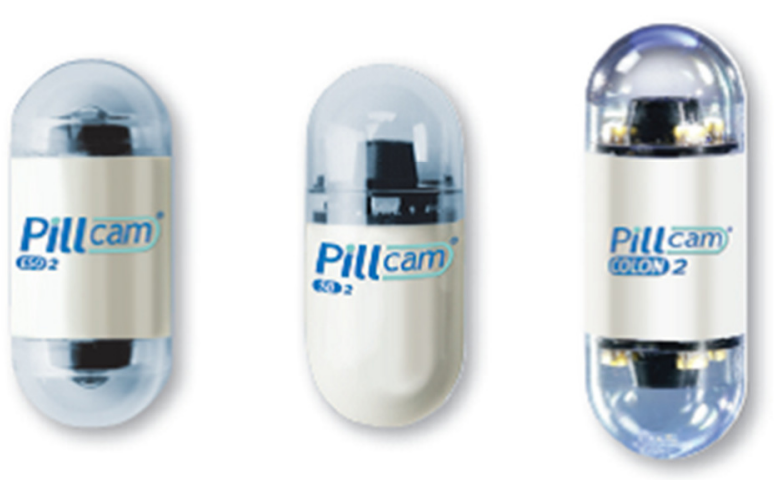

Figura 3. Diferentes modelos de la PillCam

A raíz de esto se empezaron a considerar diversas formas de manipular estas capsulas y nuevos diseños para incrementar su autonomía. Uno de estos diseños es la cápsula endoscópica diseñada para ser manipulada magnéticamente [37]. Este dispositivo se creó para tomar muestras de tejidos dentro del paciente cuando el médico lo vea necesario. Para esto se introdujo dentro de la capsula un mecanismo compuesto por dos imanes que girarán creando una compuerta que tomará la muestra, todo esto utilizando campos magnéticos. 
Basándose en lo anterior, los investigadores han concentrado sus esfuerzos en desarrollar robots médicos tipo cápsula o MCR (medical capsule robots). Estos pueden ser utilizados en otras aplicaciones médicas como intervenciones quirúrgicas. Este es el caso de una cápsula que puede liberar controladamente un clip quirúrgico para detener una hemorragia localizada en el colon. Está equipada con cuatro magnetos y un clip que será liberado por un comando externo [49]. Otros tipos de capsulas, como las híbridas (con patas o tipo submarino), se están desarrollando, y actualmente se pueden crear y desarrollar nuevos tipos de cápsulas para diversas aplicaciones médicas gracias a la reciente aparición de una plataforma de diseño de MCRs de código abierto. Esta está compuesta por una parte modular hardware con unidades genéricas de los MCRs que pueden ser añadidas, removidas o intercambiadas, además tiene un componente software con múltiples librerías y códigos ejemplo [50]. El objetivo de esta plataforma es que los ingenieros y médicos aprovechen todas las oportunidades que los mini robots tipo cápsula presentan en el campo clínico.

Como se había mencionado, otra aplicación de los mini robots en la medicina es en el área de la asistencia quirúrgica. Para lograr este objetivo se han diseñado robots que sean lo bastante pequeños para entrar en el cuerpo humano pero también de un tamaño tal que puedan ser observados y manipulados por los cirujanos. Es así como se proponen robots plegables, los cuales pueden entrar al cuerpo a través de una incisión muy pequeña u orificio natural y una vez adentro se despliegan a su tamaño original, donde podrán ser utilizados como cámara, luz o incluso pinza, y generarán mayor seguridad al cirujano y por los tanto al paciente. Uno de estos mini robots asistentes fue desarrollado por la Universidad de Nebraska, Estados Unidos, y probado in vivo. Consiste en una plataforma con un trípode plegable de $60 \mathrm{~mm}$ de alto y $15 \mathrm{~mm}$ de diámetro hecho de aluminio. Contiene una cámara y luces led, además puede girar 360 grados e inclinarse 45 grados. Su diseño está pensado para asistir cirugías laparoscópicas y el cirujano puede activarlo desde el exterior por medio de un interruptor (Figura 4) [46]. Este mismo grupo de investigación mejoró el mini robot plegable al agregarle ruedas, y posteriormente creando nuevas versiones inalámbricas [34].

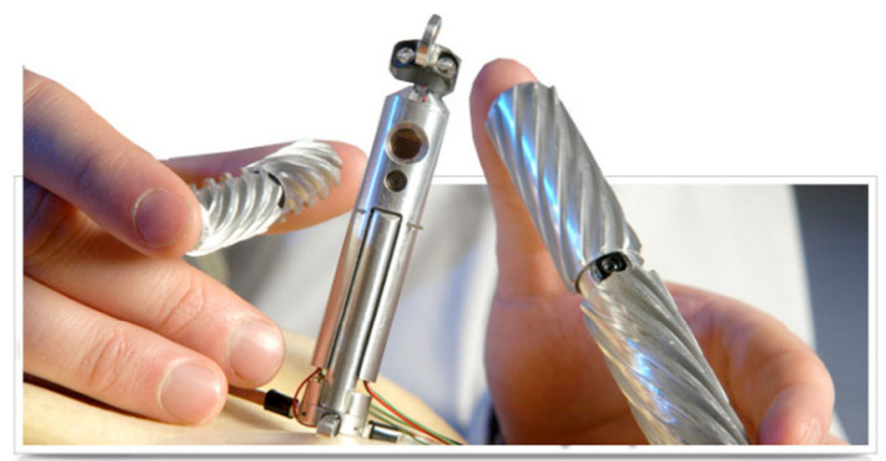

Figura 4. Robot miniatura para cirugía, Universidad de Nebraska, Estados Unidos
Otro tipo de mini robot que se puede utilizar en cirugía son aquellos del tipo modular. Como su nombre lo indica son mini robots compuestos por módulos que se ensamblan dentro del cuerpo humano y forman mini robots funcionales. Este es el caso del SMR (robot modular ingerible) que está formado por varios mini robots del tipo cápsula híbrida, los cuales son ingeridos por el paciente y a través de magnetos se conectan en el interior del cuerpo (Figura 5). Los magnetos permiten fácilmente su manipulación externa [51]. Estos mini robots modulares pueden ser utilizados en procedimientos como LESS y NOTES; para esto se han propuesto diferentes tipos de control entre los que se destaca la realización de un prototipo de mini robot un poco más grande que él, y que se ubica exteriormente para que el usuario pueda ver exactamente cuál es la forma que ha tomado el mini robot al ensamblarse, aunque esto no garantiza que tenga la misma orientación y posición que el mini robot dentro del cuerpo humano [52].

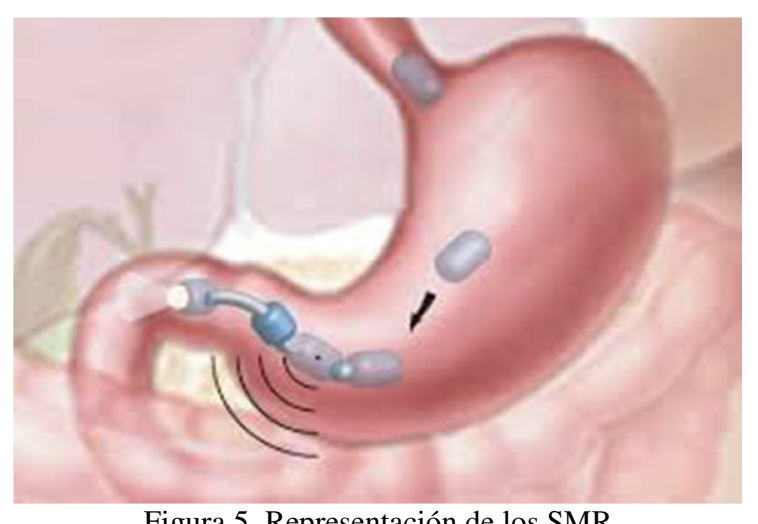

Figura 5. Representación de los SMR

Otro tipo de robots quirúrgicos son los micro robots que se utilizan para realizar cateterismos, entrega de medicamentos, procedimientos cardiacos o intervenciones intraoculares [32]. Por su menor tamaño y por el ambiente en el que se desenvuelven estos son diseñados bio miméticamente para nadar, es decir que se basan en los movimientos de renacuajos o espermatozoides [35] [36].

En este tipo de robots el principal inconveniente es la manipulación, que se dificulta por el tamaño de los dispositivos. Ante esto se han propuesto soluciones como la de accionamiento basado en electromagnetismo (EMA en inglés), la cual usa un campo electromagnético para producir fuerzas de propulsión externas, lo que facilita el movimiento, y en caso de ser necesario, la capacidad del micro robot para atravesar obstáculos usando la perforación [53] [54]. Este método se ha puesto a prueba en micro robots en forma de espiral que tienen dos magnetos, uno al inicio y otro al final, lo cual permite un mejor desempeño para potenciales aplicaciones intravasculares [55].

La combinación de estos micro robots y su manipulación electromagnética, ha dado como resultado la creación de plataformas robóticas. Estas plataformas están compuestas por grandes estructuras que generan campos electromagnéticos y ayudan a que el cirujano manipule diversos tipos de micro 
robots. Cabe destacar en este caso un controlador tele operado para manipular micro robots de movimiento híbrido [56]. Este sistema utiliza un algoritmo de control de rotación de campo magnético en ocho pasos que genera una mejor manipulación comparado con la forma tradicional de usar los campos magnéticos. Se utiliza para realizar colonoscopias con un micro robot que puede pasar de moverse en espiral a hacerlo como un pez, para que pueda adaptarse a los diferentes ambientes que hay dentro del cuerpo humano. También se ha desarrollado una plataforma para cirugía mínimamente invasiva tipo maestro-esclavo que tiene varios módulos de micro robots anclados magnéticamente. Los micro robots utilizados no tienen sensores por lo que la realimentación es visual. Se realizaron varios experimentos que mostraron que la curva de aprendizaje es corta gracias a una interfaz altamente intuitiva [57].

Comercialmente existen varios proyectos en desarrollo o ya en el mercado, como el Sensei Robotic System (Figura 6) y el Magellan Robotic System (Figura 7) de la empresa Hansen Medical. El primero se utiliza para procedimientos cardiacos y el segundo para diversos procedimientos intravasculares. Ambos son manipulados electromecánicamente y tienen una consola con pantallas y un joystick tridimensional que permite al cirujano manipular remotamente los instrumentos robóticos que están conformados por servomotores y cables con realimentación computarizada, todo montado sobre la camilla del paciente [58] [59]. El Sensei, de seis grados de libertad, ha realizado procedimientos muy similares a los convencionales por lo que se hace necesaria la realización de más pruebas para mostrar si tiene o no ventajas sobre las intervenciones más comunes. También existe el Niobe Remote Magnetic Navigation System del centro médico de la Universidad de Hackensack en Estados Unidos. Es utilizado para tratar arritmias cardiacas a través de un catéter manipulado remotamente por campos electromagnéticos (Figura 8) [60].

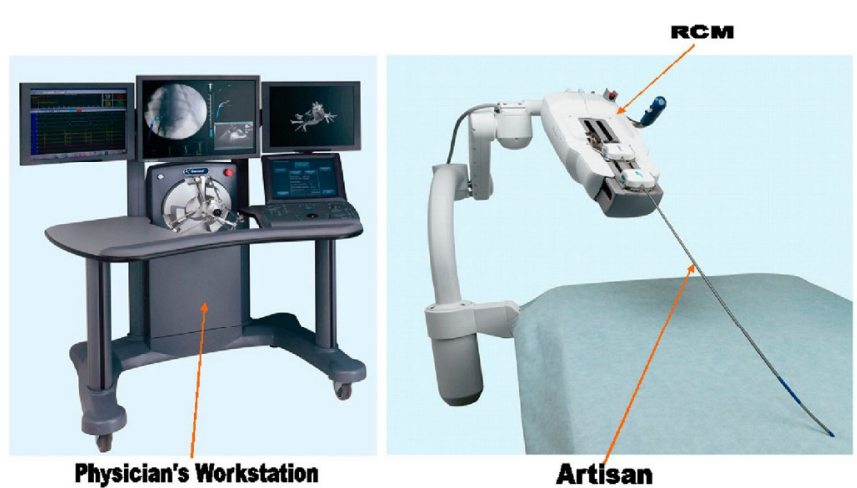

Figura 6. Sistema robótico Sensei

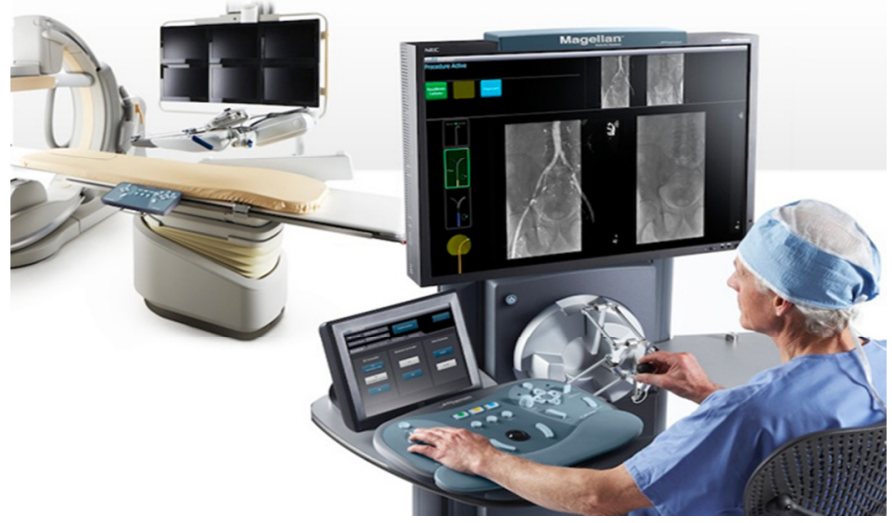

Figura 7. Sistema robótico Magellan

Estos sistemas han mejorado la estabilidad y la navegación al interior del catéter. También presentan reducción en el tiempo de los procedimientos y mayor comodidad para el cirujano. Sin embargo los procedimientos realizados con estos sistemas robóticos aún no entran al campo de la reconstrucción de tejido, esto debido al diseño limitado de los catéteres, lo cual afecta la capacidad del cirujano de maniobrar durante la cirugía [61].

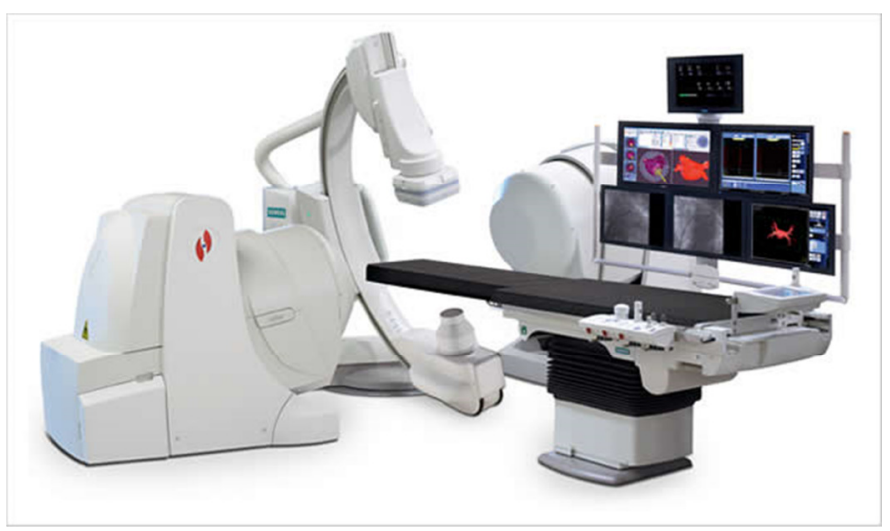

Figura 8. Navegador remoto Niobe

\section{NANO ROBOTS}

En el camino de la miniaturización los nano robots hacen parte de la tecnología más reciente. Se han diseñado para tratar tumores de difícil acceso, patologías como el cáncer (Figura 9), llevar células madre a lugares específicos o navegar dentro de la retina (Figura 10). Se espera que trabajen colaborativamente y para manipularlos se proponen varias soluciones como usar bacterias como vehículos de carga de los nano robots y manipularlos con campos electromagnéticos; usar la glucosa y el oxígeno como fuente de propulsión o, dependiendo de su composición, también manipulando la temperatura para estimular su movimiento [62]. Esto hace que detrás del diseño y construcción de estos pequeños dispositivos además de la ingeniería y la medicina estén la química y la biología, ampliando el carácter multidisciplinar de la robótica aplicada a la medicina [63]. 


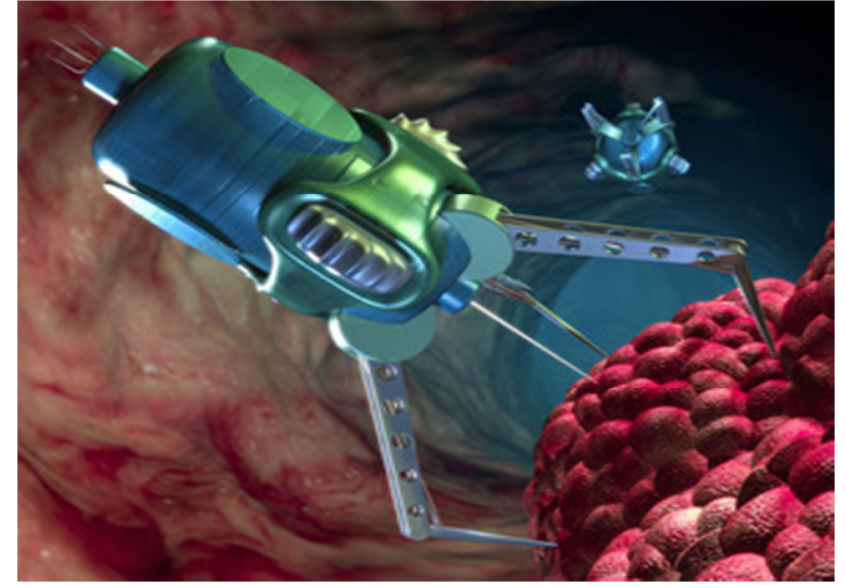

Figura 9. Interpretación de un nano robot "atacando" células cancerosas

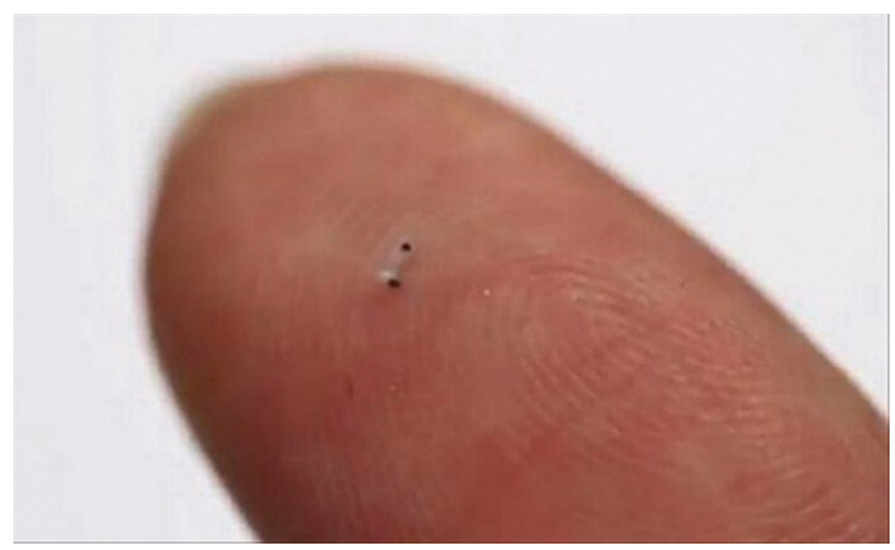

Figura 10. Muestra de un nano robot tipo almeja que puede nadar dentro de la retina

Además se han propuesto diversas soluciones con nano robots con el fin de explorar y/o tratar lugares que no se conocían o a los que no se puede acceder fácilmente. Para esto los nano robots y/o las bacterias serán cargadas con medicinas o sensores que permitan llegar a lugares remotos y adquirir todo el conocimiento posible [64]. Estos nano robots serían introducidos al cuerpo humano a través de las venas utilizando un catéter convencional.

Los avances más notables de los nano robots se darán en el tratamiento contra el cáncer. Al respecto se han simulado varios de estos pequeños dispositivos con el fin de usarlos en procedimientos quirúrgicos, esto para mostrar que la presencia de ciertas condiciones médicas y el tamaño de los vasos sanguíneos pueden interferir en la actuación del nano robot [65]. Esto ayudará a los cirujanos a prever situaciones que se puedan presentar cuando en el futuro se realicen este tipo de procedimientos.

Entre las ventajas del uso de la nano robótica en la medicina se encuentran el bajo costo de los procedimientos, recuperación rápida, cirugías no invasivas, capacidad para alcanzar lugares remotos a los que el cirujano no puede acceder, y mayor exactitud en los procedimientos [66] [62]. Sin embargo también presenta desventajas como que su diseño inicial es muy costoso, diseñarlos es complejo y pueden presentar problemas al ser afectados por interferencia eléctrica [62].

Se espera que para el año 2020 se inicie la era de la nano robótica, ya que la expectativa es que se podrán diseñar y construir todo tipo de dispositivos nano métricos autónomos, con sus componentes y computadores que podrán usarse en cirugía [67].

\section{CONCLUSIONES}

La cirugía robótica ha tenido un avance vertiginoso en la última década, lo que ha decantado en ventajas tanto para cirujanos como para pacientes. El robot quirúrgico más destacado hasta la fecha es el Da Vinci Surgical System gracias al cual Intuitive Surgical ha tenido el monopolio del negocio de la cirugía robótica en el mundo. Si bien, en los últimos años varias empresas han desarrollado otros robots quirúrgicos que pronto entrarán al mercado y permitirán que la cirugía robótica llegue a más usuarios y que más procedimientos puedan realizarse con estos equipos.

El principal inconveniente de los grandes asistentes quirúrgicos son los elevados precios que tienen, lo que hace que muchos pacientes y cirujanos opten por continuar con la realización de procedimientos tradicionales. Además su gran tamaño los hace poco prácticos para algunas intervenciones delicadas como la cirugía cardiaca o procedimientos en niños.

Se espera que la investigación y difusión del conocimiento sobre los asistentes robóticos sea amplia y pueda convencer a médicos y pacientes sobre las ventajas de estos y cómo han sido desarrollados para servir de apoyo y mejorar las capacidades del médico, no para reemplazarlo. Para esto, es ideal el uso de los mini robots que han sido diseñados de tal forma que sean más sencillos de manipular, ya que al ser menos invasivos generan más confianza en el equipo médico y en los pacientes. Estos han surgido como una opción a los grandes asistentes pues su costo es menor y son ideales para realizar procedimientos como LESS y NOTES pues su tamaño pequeño corrige varios de los inconvenientes de estos procedimientos; además la curva de aprendizaje es más corta.

Los campos electromagnéticos han resultado ser la mejor solución a uno de los principales problemas de los mini y micro robots: la manipulación. El uso de estos campos ha permitido diseñar y mejorar diferentes tipos de micro robots como los robots tipo cápsula o los robots modulares, además con esto se amplió la gama de instrumentos que pueden ser cargados por un mini robot como pinzas, cámaras, luces o cauterizadores.

Los nano robots para uso médico se han planteado como el futuro de la medicina y sus ventajas son múltiples al igual que sus usos tanto en cirugía como en investigación. Un nano robot podría ayudar al cirujano a realizar procedimientos en lugares que normalmente no podría alcanzar, además de adquirir información privilegiada de la situación al interior del 
paciente. Sin embargo, las investigaciones sobre este tema, en su mayoría, solo son simuladas y no pasan de diseños y propuestas

Aunque Colombia cuenta con dos robots Da Vinci, estos no se han utilizado para investigación, solo para procedimientos o entrenamiento, lo que hace que la investigación y desarrollo de la robótica quirúrgica en el país esté a manos exclusivamente de los diferentes grupos de investigación en las universidades. Pero esto no ha sido inconveniente para avanzar en diversos proyectos y ser un referente de este tema en la región.

\section{REFERENCIAS}

[1] D. Ruiz, V. Pérez, M. Betancur, J. Bustamante, "Cirugía robótica mínimamente invasiva: análisis de fuerza y torque", Revista Ingeniería Biomédica, Vol. 4, No. 8, pp. 84-92, 2010.

[2] P. Ricci, R. Lema, V. Solà, J. Pardo, E. Guiloff, "Desarrollo de la cirugía laparoscópica: pasado, presente y futuro. Desde hipócrates hasta la introducción de la robótica en laparoscopia ginecológica", Revista chilena de Obstetricia y Ginecología, Vol. 73, No. 1, pp. 63-75, 2008.

[3] J.D. Raman, J.A. Cadeddu, P. Rao, A. Rane, "Single-incision laparoscopic surgery: initial urological experience and comparison with natural-orifice transluminal endoscopic surgery", BJU International, Vol. 101, No. 12, pp. 1493-1496, 2008.

[4] J.F. Noguera Aguilar, C. Moreno Sanz, A. Cuadrado García, J.M. Olea Martínez-Mediero, R. Morales Soriano, J.C. Vicens Arbona, M.L. Herrero Bogajo, L. Lozano Salva, "NOTES. Historia y situación actual de la cirugía endoscópica por orificios naturales en nuestro país", Cirugía Española, Vol. 88, No. 4, pp. 222-227, 2010.

[5] T. González León, E. Rodríguez Verde, A. Núñez Roca, "Consideraciones sobre la cirugía endoscópica transluminal a través de un orificio natural", Revista Cubana de Medicina Militar, Vol. 40, pp. 34, 2011.

[6] G. Yin, W.K. Han, S. Faddegon, Y.K. Tan, Z.W. Liu, E.O. Olweny, D.J. Scott, J.A. Cadeddu, "Laparoendoscopic Single Site (LESS) In Vivo Suturing Using a Magnetic Anchoring and Guidance System (MAGS) Camera in a Porcine Model: Impact on Ergonomics and Workload", Urology, Vol.81, pp.80-84, 2013.

[7] YS. Kwoh, J. Hou , EA. Jonckheere, S. Hayati, "A robot with improved absolute positioning accuracy for CT guided stereotactic brain surgery", IEEE Transactions on Biomedical Engineering, Vol. 32, No. 2, pp. 153$160,1988$.

[8] S. J. Harris , F. Arambula-Cosio, Q. Mei, R. D. Hibberd, B. L. Davies, J. E. Wickham, M. S. Nathan, B. Kundu, "The Probot - an active robot for prostate resection", Journal of Engineering in Medicine, Vol. 211, No. 4, pp.317-325, 1997.

[9] H. A. Paul, W. L. Bargar, B. Mittlestadt, B. Musits, R. H. Taylor, P. Kazanzides, J. Zuhars, B. Williamson, W. Hanson, "Development of a surgical robot for cementless total hip arthroplasty", Clinical Orthopaedics \& Related Research, Vol. 285, pp. 57-66, 1992.

[10] M. Diana, J. Marescaux, "Robotic Surgery" British Journal of Surgery, Vol. 102, No. 2, pp. 15-28, 2015.

[11] A. Carbajal, "Cirugía Robótica", Cirujano General, Vol. 24, No. 4, pp. 314-320, 2003.

[12] S. Unger, H. Unger, R. Bass, "AESOP robotic arm", Surgical Endoscopy, Vol. 8, No. 9, pp. 1131-1131, 1994.

[13] H. Reichenspurner, R. J. Damiano, M. Mack, D. H. Boehm, H. Gulbins, C. Detter, B. Meiser, R. Ellgass, B. Reichart, "Use of the voicecontrolled and computer-assisted surgical system Zeus for endoscopic coronary artery bypass grafting", The Journal of Thoracic and Cardiovascular Surgery, Vol.118, No. 1, pp. 11-16, 1999.

[14] G. Ballantyne, F. Molle, "The Da Vinci telerobotic surgical system: the virtual operative field and telepresence surgery",Surgical Clinics of North America, Vol.83, pp. 1293-1304, 2003.

[15] R. Valero, Y.H. Ko, S. Chauhan, O. Schatloff, A. Sivaraman, R.F. Coelho, F. Ortega, K.J. Palmer, R. Sanchez-Salas, X. Cathelineau, V.R. Patel, H. Davila, "Cirugía Robótica: historia e impacto en la enseñanza", Actas Urológicas Españolas, , Vol. 35, No. 9, pp. 540-545, 2011.
[16] SPORT Surgical System. Disponible: http://www.titanmedicalinc.com/product/. Acceso octubre de 2015.

[17] S. Gidaro, E. Altobelli, C. Falavolti, A. M Bove, E. M Ruiz, M. Stark, G. Ravasio, S. S. Lazzaretti, B. Maurizio, "Vesicourethral anastomosis using a novel telesurgical system with haptic sensation, the Telelap AlfX: a pilot study”, Surgical Technology International, Vol. 24, pp. 35-40, 2014.

[18] U. Hagn , R. Konietschke, A. Tobergte, M. Nickl, S. Jörg, B. Kübler, G. Passig, M. Gröger, F. Fröhlich, U. Seibold, L. Le-Tien, A. AlbuSchäffer, A. Nothhelfer, F. Hacker, M. Grebenstein, G. HirzingerShow less, "DLR MiroSurge: a versatile system for research in endosopic telesurgery", International Journal of Computer Assisted Radiology and Surgery, Vol. 5, No. 2, pp. 183-193, 2010.

[19] A. Tobergte, P. Helmer, U. Hagn, P. Rouiller, S. Thielmann, S. Grange, A. Albu-Schaffer, F. Conti, G. Hirzinger, "The sigma.7 haptic interface for MiroSurge: A new bi-manual surgical console", 2011 IEEE/RSJ International Conference on Intelligent Robots and Systems, San Francisco, CA, USA, 2011.

[20] SOFIE. Disponible: https://www.tue.nl/en/research/researchinstitutes/robotics-research/projects/sofie/. Acceso octubre de 2015.

[21] Eindhoven University of Technology. Better surgery with new surgical robot with force feedback. Disponible: http://www.sciencedaily.com/releases/2010/09/100928083848.htm. Acceso octubre de 2015.

[22] E.J. Prendes-Sillero, J.M. Díaz-Pavón, F. de la Portilla-de Juan, "Cirugía robótica y cáncer de recto", Revista Andaluza de Patología Digestiva, Vol. 37, No. 2, pp. 74-80, 2014.

[23] Cirugía robótica, clínica Marly. Disponible: http://www.marly.com.co/serv_cirugia_robotica.html. Acceso octubre de 2015.

[24] Sala de cirugía robótica en Da Vinci. Fundación INSIMED. Disponible: http://www.insimed.org/index.php/ES-es/instalaciones/83-sala-cirugiaroboticaen-da-vinci. Acceso octubre de 2015.

[25] J. D. Posada, J. V. Padilla, M. D. Castillo, S. Molina, "Control de un brazo robótico usando el hardware kinect ${ }^{\circledR}$ de microsoft", Prospectiva, Vol. 11, No. 2, pp. 88-93, 2013.

[26] S. Salinas, A. Vivas, "Modelado, simulación y control del robot para cirugía laparoscópica LapBot", Revista Chilena de Ingeniería, Vol. 17, No. 3, pp. $317-328,2009$.

[27] V. Torres, C. Méndez, A. Vivas, S. Salinas, "Diseño y simulación en 3D de un robot porta endoscopio para operaciones de laparoscopia", $V$ Seminario Internacional de Ingeniería Electrónica, Bucaramanga, Colombia, 2011.

[28] D. Vera, A. Vivas, "Ambiente virtual para el entrenamiento de cirugías laparoscópicas utilizando robots", Panamerican Health Care Exchanges, Medellín, Colombia, 2013.

[29] J. Genoy, L. Rodríguez Ortiz, S. Salinas, "Interfaz háptica de cuatro grados de libertad para aplicaciones quirúrgicas", Revista Ingeniería Biomédica, Vol. 5, No. 9, pp. 35-42, 2011.

[30] M. Díaz, J. Escobar, A. Vivas, "Interfaz háptica tipo guante con realimentación vibratoria", Revista EIA, Vol. 12, No. 23, pp. 29-39, 2015.

[31] J. Hurtado, A. Nástar, A. Vivas, "Sistema de captura de gestos con KINECT para la manipulación de robots quirúrgicos virtuales", ITECKNE, Vol. 12, No. 1, pp. 17-24,2014.

[32] S. Martel, "Journey to the center of a tumor", Spectrum IEEE, Vol. 49, pp. 48-53, 2012.

[33] R. Bogue, "Miniature and microrobots: a review of recent developments", Industrial Robot: An International Journal, Vol. 42, No. 2, pp.98-102, 2015.

[34] A. Forgione, "In vivo minirobots for natural orifice transluminal surgery. Current status and future perspectives", Surgical Oncology, Vol.18, pp. 121-129, 2009.

[35] B. Chen, Y. Liu, S. Chen, S. Jiang, H. Wu, "A Biomimetic Spermatozoa Propulsion Method for Interventional Mini Robot", Journal of Bionic Engineering Suppl. Vol. 5, pp. 106-112, 2008

[36] K. Tokida, A. Yamaguchi, K. Takemura, S. Yokota, K. Edamura, "A BioInspired Robot Using Electro-Conjugate Fluid", Journal of Robotics and Mechatronics Vol. 25, pp. 16-24, 2013.

[37] M. Simi, G. Gerboni, A. Menciassi, P. Valdastri, "Magnetic Mechanism for Wireless Capsule Biopsy", Journal of Medical Devices, Vol. 6, pp. 017611-017611-1, 2012.

[38] S.L. Best, W. Kabbani, D.J. Scott, R. Bergs, H. Beardsley, R. Fernandez, L.B. Mashaud, J.A. Cadeddu, "Magnetic Anchoring and Guidance System Instrumentation for Laparo-endoscopic Single-site 
Surgery/Natural Orifice Transluminal Endoscopic Surgery: Lack of Histologic Damage After Prolonged Magnetic Coupling Across the Abdominal Wall", Urology, Vol. 77, No 1, pp. 243-247, 2011.

[39] S. Uenoa, K. Takemura, S. Yokota, K. Edamura, "Mini inchworm robot using electro-conjugate fluid", Sensors and Actuators A: Physical, Vol. 216, pp. $36-42,2014$.

[40] J.D. Raman, D.J. Scott, J.A. Cadeddu, "Role of Magnetic Anchors During Laparoendoscopic Single Site Surgery and NOTES", Journal of Endourology, Vol. 23, No 5, pp. 781-786, 2009.

[41] S. Tognarelli, M. Salerno, G. Tortora, C. Quaglia, P. Dario, A. Menciassi, "An Endoluminal Robotic Platform for Minimally Invasive Surgery", The Fourth IEEE RAS/EMBS International Conference on Biomedical Robotics and Biomechatronics, Roma, Italy. June 24-27, 2012.

[42] B.S. Terry, Z.C. Mills, J.A. Schoen, M.E. Rentschler, "Single-PortAccess Surgery with a Novel Magnet Camera System", IEEE Transactions on Biomedical Engineering, Vol. 59, No 4, pp. 1187-1193, 2012.

[43] J.C. Kuo, H.W. Huang, S.W. Tung, Y.J. Yang, "A hydrogel-based intravascular minigripper manipulated using magnetic fields", Sensors and Actuators A: Physical, Vol. 211, pp. 121-130, 2014.

[44] J. Cadeddu, R. Fernandez, C. Tracy, M. Desai, S.-J. Tang, P. Rao, M. Desai, D. Scott, "Novel magnetically guided intra-abdominal camera to facilitate laparoendoscopic single-site surgery: initial human experience", Surgical Endoscopy, Vol. 23, pp. 1894-1899, 2009.

[45] N.A. Arain, D.C. Hogg, R. Bergs, L. Rondon, J.A. Cadeddu, R. Fernandez, D.J. Scott, "Magnetically anchored camera and percutaneous instruments maintain triangulation and improve cosmesis compared with single-site and conventional laparoscopic cholecystectomy", Surgical Endoscopy, Vol. 26, pp. 3457-3466, 2012.

[46] D. Oleynikov, M. Rentschler, A. Hadzialic, "Miniature robots can assist in laparoscopic cholecystectomy", Surgical Endoscopy, Vol. 19, pp.473476, 2005.

[47] R. Eliakim, K. Yassin, I. Shlomi, A. Suissa, G.M. Eisen, "A novel diagnostic tool for detecting oesophageal pathology: the PillCam oesophageal video capsule", Alimentary Pharmacology \& Therapeutics, Vol. 20, No. 10, pp. 1083-1089, 2004.

[48] Y.C Metzger, S.N Adler, A.B. Shitrit, B. Koslowsky, I. Bjarnason, "Comparison of a new PillCam ${ }^{\mathrm{TM}} \mathrm{SB} 2$ video capsule versus the standard PillCam ${ }^{\mathrm{TM}}$ SB for detection of small bowel disease", Reports in Medical Imaging, Vol.2, pp.7-11, 2009.

[49] P. Valdastri, C. Quaglia, E. Susilo, A. Menciassi, P. Dario, C.N. Ho, G. Anhoeck, M.O. Schurr, "Wireless therapeutic endoscopic capsule: in vivo experiment", Endoscopy, Vol. 40, No. 12, pp. 979-982, 2008.

[50] M. Beccani, H. Tunc, A. Taddese, E. Susilo, P. Volgyesi, A. Ledeczi, P. Valdastri, "Systematic Design of Medical Capsule Robots", IEEE Design \& Test, Vol. 32, No. 5, pp. 98-108, 2015.

[51] Z. Nagy, R. Oung, J. J. Abbott, B. J. Nelson, "Experimental Investigation of Magnetic Self-Assembly for Swallowable Modular Robots", IEEE/RSJ International Conference on Intelligent Robots and Systems, Niza, Francia, 2008.

[52] A. Zygomalas, K. Giokas, D. Koutsouris, "In Silico Investigation of a Surgical Interface for Remote Control of Modular Miniature Robots in Minimally Invasive Surgery", Minimally Invasive Surgery, Vol, 2014, pp. 1-5, 2014.

[53] H. Choi, K. Cha, J. Choi, S. Jeong, S. Jeon, J. Parka, S. Parka, G. Jang, "EMA system with gradient and uniform saddle coils for 3D locomotion of microrobot", Sensors and Actuators A: Physical, Vol. 163, pp. 410$417,2010$.

[54] C. Yu, J. Kim, H. Choi, J. Choi, S. Jeong, K. Cha, J. Park, S. Park, "Novel electromagnetic actuation system for three-dimensional locomotion and drilling of intravascular microrobot", Sensors and Actuators A: Physical, Vol. 161, pp. 297-304, 2010.

[55] S. Jeong, H. Choi, K. Cha, J. Li, J. Park, S. Park, "Enhanced locomotive and drilling microrobot using precessional gradient magnetic field", Sensors and Actuators A: Physical, Vol. 171, pp. 429-435, 2011.

[56] J. Guo, S. Guo, X. Wei, "A Novel Tele-operation Controller for wireless Microrobots in-pipe with Hybrid Motion", Robotics and Autonomous Systems, por publicar, 2015.

[57] I. Rivas-Blanco, V. F. Muñoz, I. García-Morales, G. Tortora, A. Menciassi, "Arquitectura de Control de una Plataforma Robótica sin Realimentación de Posición Articular para Cirugía Mínimamente Invasiva”, XXXVI Jornadas de Automática, Bilbao, España, 2015.
[58] W. Saliba, V. Y. Reddy, O. Wazni, J, E. Cummings, J. D. Burkhardt, M. Haissaguerre, J. Kautzner, P. Peichl, P. Neuzil, V. Schibgilla, G. Noelker, J. Brachmann, L. Di Biase, C. Barrett, P. Jais, A. Natale, "Atrial Fibrillation Ablation Using a Robotic Catheter Remote Control System. Initial Human Experience and Long-Term Follow-Up Results", Journal of the American College of Cardiology, Vol. 51, No. 25, pp. 2407-2411, 2008.

[59] R. P. Cambria, "Clinical applications of robotic technology in vascular and endovascular surgery", Journal of Vascular Surgery, Vol. 53, pp. 493-499, 2011.

[60] S. Ernst, F. Ouyang, C. Linder, K. Hertting, F. Stahl, J. Chun, H. Hachiya, D. Bänsch, M. Antz, K. Kuck, "Initial Experience With Remote Catheter Ablation Using a Novel Magnetic Navigation System Magnetic Remote Catheter Ablation", Circulation, Vol.109, pp. 1472$1475,2004$.

[61] N. V, Vasilyev, P. E. Dupont, P. J del Nido, "Robotics and imaging in congenital heart surgery", Future Cardiology, Vol. 8, No. 2, pp. 285296, 2012.

[62] P. Khulbe, "Nanorobots: a review", International Journal of Pharmaceutical Sciences and Research, Vol. 5, No. 6, pp. 2164-2173, 2014.

[63] C. Bergeles, G.Z Yang, "From Passive Tool Holders to Microsurgeons: Safer, Smaller, Smarter Surgical Robots", IEEE Transactions on Biomedical Engineering, Vol. 61, No. 5, pp.1565-1576, 2014.

[64] S. Martel, O. Felfoul, J. Mathieu, A. Chanu, S. Tamaz, M. Mohammadi, M. Mankiewicz, N. Tabatabaei, "MRI-based Medical Nanorobotic Platform for the Control of Magnetic Nanoparticles and Flagellated Bacteria for Target Interventions in Human Capillaries", The International Journal of Robotics Research, Vol. 28, No. 9, pp. 1169$1182,2009$.

[65] A. Cavalcanti, B. Shirinzadeh, D. Murphy, J. A. Smith, "Nanorobots for Laparoscopic Cancer Surgery", IEEE ICIS 2007 International Conference on Computer and Information Science, Melbourne, Australia, 2007.

[66] A.S Bath, "Nanobots: the Future of Medicine", International Journal of Engineering and Managment Sciences, Vol. 5, No. 1, pp. 44-49, 2004.

[67] R. A. Freitas Jr, "Nanotechnology, nanomedicine and nanosurgery", Journal of Surgery, Vol. 3, pp.243-246, 2005. 